\title{
Use of computed tomography and mechanical CPR in cardiac arrest to confirm pulmonary embolism: a case study
}

\author{
Elaine Catrin Schubert*; Karl-Georg Kanz, PhD, $\mathrm{MD}^{\dagger}$; Ulrich Linsenmaier, PhD, MD*; \\ Viktoria Bogner, MD"; Stefan Wirth, $\mathrm{MD}^{\ddagger}$; Matthias Angstwurm, PhD, MD"
}

\section{ABSTRACT}

Precise therapeutic decision-making is vital in managing out-of-hospital cardiac arrest. We present an interesting approach where suspected pulmonary embolism could be confirmed by early computed tomography in cardiac arrest. Chest compressions were performed automatically by mechanical devices also during the acquisition of computed tomography data and subsequent thrombolysis.

\section{RÉSUMÉ}

La précision des décisions relatives au traitement est d'importance « vitale » dans la prise en charge des arrêts cardiaques extrahospitaliers. Sera présentée ici une intervention intéressante, qui a permis de confirmer, par une tomodensitométrie (TDM) précoce, une embolie pulmonaire présumée chez une patiente en état d'arrêt cardiaque. Les compressions thoraciques, réalisées automatiquement par un appareil mécanique se sont poursuivies durant l'acquisition des données de la TDM et le traitement thrombolytique qui s'en est suivi.

Keywords: Out of hospital Cardiac Arrest (OHCA), Pulmonary Embolism, In-field Thrombolysis, Automated Chest Compression Devices, Computed Tomography (CT)

\section{INTRODUCTION}

A common cause of out-of-hospital cardiac arrest (OHCA) requiring cardiopulmonary resuscitation (CPR) is thrombus formation resulting in coronary artery occlusion or pulmonary embolism. ${ }^{1}$ In order to save the lives of those affected, the cause needs to be identified as soon as possible in order to start the targeted therapy. This process begins with the advanced cardiac life support (ACLS). Thrombolysis in the field can be considered in certain situations. The European Resuscitation Council (ERC) 2010 guidelines do not recommend this in all cases of cardiac arrest, however thrombolytics can be considered when pulmonary embolism is highly suspected or proven in order to improve survival and neurological outcome of patients. ${ }^{1}$ In addition to thrombolysis, an active mechanical guide wire fragmentation of the thrombus with a flexible endovascular wire is effective in improving pulmonary perfusion and clinical outcome. ${ }^{2}$

In OHCA, optimal CPR is vital for the survival of the patients. ${ }^{3}$ Effective chest compressions with a depth of at least $38 \mathrm{~mm}$ and maintenance of a high rate are most relevant for the quality of CPR. ${ }^{4}$ This may sometimes be difficult to implement in all environments with manual chest compressions. Using automated chest compression devices may help to ensure a continuous good performance of chest compressions and perfusion throughout the treatment, transport, and movement of patients. It can be used during further diagnositic testing such as computed tomography (CT). ${ }^{1,5}$ Early CT in these situations has demonstrated improved survival rates. ${ }^{6}$

\section{CASE REPORT}

A 26-year-old female presented at a general practitioner's surgery with dyspnoea. Whilst obtaining history the patient arrested. CPR was initiated immediately by the

From the *Department of Clinical and Interventional Radiology, and tDepartment of Trauma Surgery, Klinikum rechts der Isar, Technische Universität München, Munich, Germany; ¥Department of Clinical Radiology, ףDepartment of Surgery and ॥Department of Internal Medicine, University Hospital, Campus Innenstadt, Ludwig-Maximilians-Universität, Munich, Germany.

Correspondence to: Dr. Kanz, Klinikum rechts der Isar, Technical University of Munich, Department of Trauma Surgery, Ismaninger Straße 22, 81675 Munich, Germany; Email: prof.kanz@tum.de 


\begin{tabular}{|c|c|c|}
\hline \multirow[t]{12}{*}{ Day 01} & $-:-$ & Dyspnea, collapse in practitioner's surgery \\
\hline & & BLS by present staff \\
\hline & 11:07 & EMS activated \\
\hline & 11:08 & EMS dispatched \\
\hline & $11: 12$ & Arrival of First Responder Fire Engine; AED no shock advised; mCPR \\
\hline & $11: 14$ & Arrival of ambulance physician; GCS 3; endotracheal intubation, adrenaline endotracheally/IV, thrombolysis IV \\
\hline & $11: 28$ & Arrival of shock trauma unit under mCPR \\
\hline & $11: 32$ & Contrast-enhanced MDCT study; pulmonary embolism of both sides \\
\hline & $11: 50$ & Intermittent ROSC with bradycardia \\
\hline & $12: 05$ & Blood pressure 85/50 mm Hg under vasopressor support \\
\hline & $12: 15$ & Intraluminar mechanical thrombus fragmentation \\
\hline & $12: 20$ & Stable blood pressure 103/55 mm Hg with heart rate 95/min Admission to ICU \\
\hline Day 02 & \multicolumn{2}{|r|}{$\begin{array}{l}\text { Abdominal compartment syndrome; decompression laparotomy, double-loop ileostomy, temporary abdominal closure by } \\
\text { absorbable mesh }\end{array}$} \\
\hline Day 09 & \multicolumn{2}{|r|}{ Segmental colon ischemia, hemicolectomy, abdominal closure } \\
\hline Day 40 & \multicolumn{2}{|r|}{ Discharge from hospital; no neurological deficit } \\
\hline Day 112 & \multicolumn{2}{|r|}{ Closure of ileostomy and colostomy; normal colonic passage } \\
\hline
\end{tabular}

presiding staff and emergency medical services (EMS) activated at min 0 (Table 1). The First Responder Unit was dispatched and arrived at min 5. Manual CPR was continued and an automated external defibrillator (AED Pro, Zoll, Chelmsford, USA) was applied. There was no shock recommendation given by the device.

Mechanical CPR (mCPR) was continued by the automated chest compression device LUCAS ${ }^{\circledR}$ (Jolife, Lund, Sweden). A physician-staffed ambulance reached the scene shortly after, at min 7 . The patient remained pulseless. The initial electrocardiography (ECG) showed a systole and intermittent bradycardia with a rate of less than 30 beats/min, resulting in a pulseless electrical activity (PEA). The patient was endotracheally intubated by the physician and $3 \mathrm{mg}$ adrenaline (epinephrine) were given via the tube. Intravenous access was obtained via a foot vein and $1 \mathrm{mg}$ adrenaline was administered every $5 \mathrm{~min}$, as well as a dose of $80 \mathrm{IU}$ vasopressin.

Pulmonary embolism was the highly suspected diagnosis given the history of dyspnoea prior to collapse, knee brace after surgery, and intake of oral contraceptives. Rescue-in-field thrombolysis was initiated therefore by the EMS physician with $50 \mathrm{mg}$ alteplase (Actilyse Boehringer Ingelheim $\mathrm{GmbH}$, Ingelheim am Rhein, Germany). In addition, $5000 \mathrm{IU}$ unfractionated heparin were given.

Under ongoing mCPR, the patient was transported to our shock trauma unit with integrated CT and arrived at $\min 21$. The correct position of the tube was

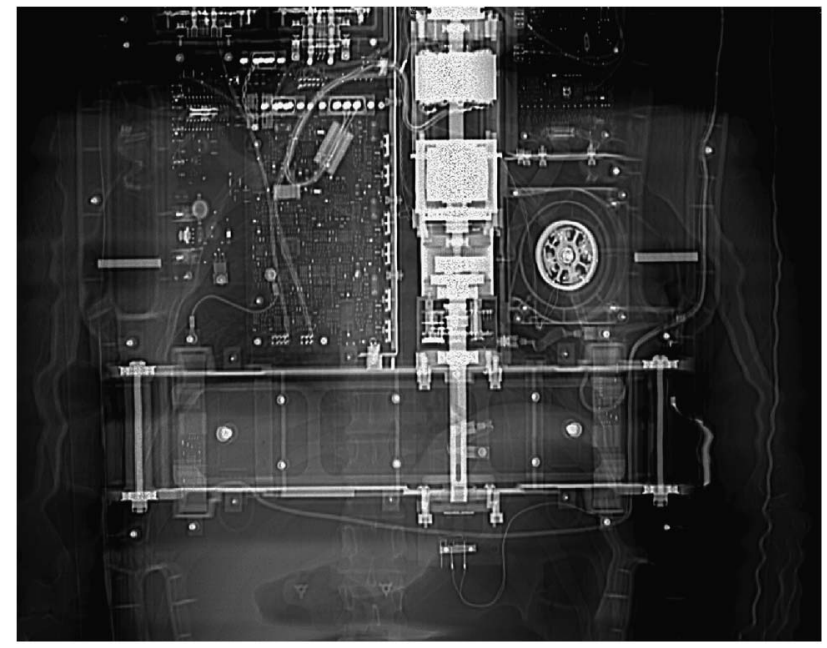

Figure 1. Scout scan with running AutoPulse ${ }^{\circledR}$ device.

confirmed by $\mathrm{ETCO}_{2}$ of $20 \mathrm{~mm} \mathrm{Hg} ; \mathrm{SpO}_{2}$ was $68 \mathrm{~mm}$ $\mathrm{Hg}$ with $\mathrm{FiO}_{2}$ of 1.0.

A change of the automated chest compression device to the AutoPulse ${ }^{\circledR}$ device (Zoll Medical GmbH, Chelmsford, MA, USA) was necessary as LUCAS devices do not fit into the gantry of our CT due to their height. At min 25 the multidetector CT imaging was initiated in a 64-slice CT scanner (Light Speed VCT, GE Healthcare, Waukesha, Wisconsin, USA). A scout scan was performed while running the AutoPulse device (Figure 1). In order to do this, we administered a contrast bolus IV, followed by two minutes of automated chest compressions for distribution. The mCPR 


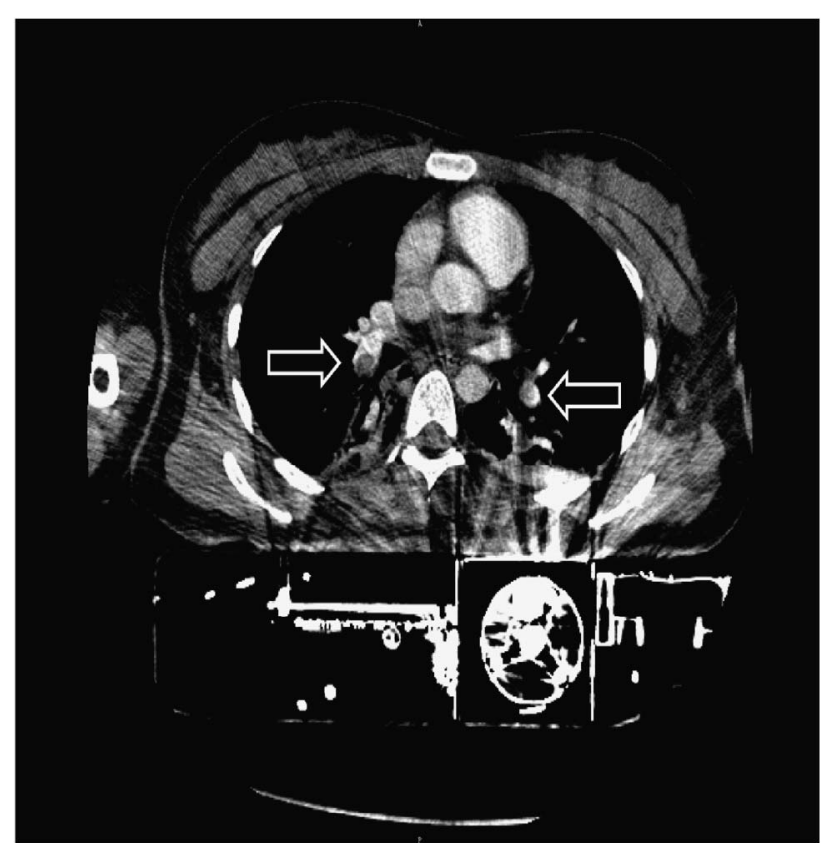

Figure 2. Contrast enhanced chest CT showing central pulmonary embolism of both sides (arrows).

device was put on hold shortly, whilst the whole body CT was performed. Contrast-enhanced CT of the chest showed a central pulmonary embolism of both sides (Figure 2).

Systemic thrombolysis was continued. At min 58 the ECG showed a systole with intermittent bradycardic episodes at a rate of $30 / \mathrm{min}$ and a blood pressure of 85/ $50 \mathrm{~mm} \mathrm{Hg}$, still under vasopressor support.

In the angiography suite, the thrombus was fragmented on both sides using a Terumo ${ }^{\circledR}$ endovascular flexible wire (Terumo Europe N.V. Interleuvenlaan 40B-3001 Leuven, Belgium) via a jugular vein access at $\min 68$.

Immediately after the procedure the blood pressure rose to $103 / 55 \mathrm{~mm} \mathrm{Hg}$ and the vasopressor support was reduced.

During intensive care unit (ICU) treatment the patient suffered from several complications. She developed systemic inflammatory response syndrome (SIRS) and disseminated intravascular coagulation (DIC) with multiple organ dysfunction syndrome, including renal failure. On day 2, due to an acute abdominal compartment syndrome, a decompression laparotomy and double-loop ileostomy were performed. On day 9, a hemicolectomy of the right colon and a single-loop transversostomy was necessary due to segmental colon ischemia. Part of the small intestine was resected and an ileostomy accomplished.
The patient recovered with no neurological deficit and was discharged from hospital at day 40. At day 112 the ileostomy and the colostomy could both be closed and a normal colonic passage was reconstituted.

\section{DISCUSSION}

As stated in the ERC 2010 guidelines, ongoing CPR is not a contraindication for fibrinolysis. ${ }^{1}$ Although not all cases of cardiac arrest should undergo fibrinolysis in the field, the ERC 2010 guidelines suggest it should be considered when pulmonary embolism is highly suspected in the field and proven in hospital. ${ }^{1}$ The TROICA-study ${ }^{7}$ was designed to assess safety and efficiency of prehospital thrombolytic therapy with tenecteplase versus placebo in cardiac arrest, and is one of the largest trials, with 1,050 enrolled patients. The results did not show an improvement in 30-day survival for all patients, but cases with pulmonary embolism did benefit from early thrombolysis. ${ }^{8}$

In a smaller case series and retrospective analysis of patients with pulmonary embolism undergoing CPR, ${ }^{9}$ the patient outcomes support thrombolytic therapy when pulmonary embolism is highly suspected. Our patient had known risk factors, including a history of knee surgery with immobilisation, as well as oral contraceptive use. Most importantly, her main symptom prior to cardiac arrest was dyspnoea. The pulmonary embolism was proven later in our shock trauma unit by early contrast-enhanced CT. In addition, we performed an active fragmentation of the embolism (which studies suggest may improve circulation and recovery of patients ${ }^{2}$.

During manual CPR there can be negative effects of rescuer fatigue, resulting in ineffective chest compressions. ${ }^{10}$ In an uncontrolled clinical setting there may even be frequent interruptions of the compressions. ${ }^{1,11}$ For our patient, CPR was performed from the beginning with $\mathrm{mCPR}$ devices at a constant rate and depth. The ERC 2010 guidelines state that during an ambulance transfer and movement of patients, mCPR devices may help to maintain a good quality of CPR. ${ }^{1}$ With $\mathrm{mCPR}$, there is no interruption of the compressions in the process of further examination in the hospital, as shown in an initial case series. ${ }^{5}$

The PROCAT study examined the benefit of an early imaging algorithm in an ICU database of OHCA with return of spontaneous circulation (ROSC). Coronary angiography was performed unless the 
patient had neurological or respiratory signs, in which case a chest CT was done. In this manner nearly twothirds of the cases were diagnosed, leading to targeted therapy. ${ }^{12}$ The feasibility of early imaging with focused CT in resuscitation (FACTR) has been discussed in other reports. ${ }^{13,14}$

During cardiac arrest there is no circulation for contrast-enhanced CT imaging. The use of mCPR devices when performing a CT seems to be valuable. ${ }^{5}$ Those devices are able to provide a blood flow and therefore also the distribution of the contrast solution when used during CT image acquisition. The ERC 2010 guidelines state that mCPR in cardiac arrest may be used effectively whilst undergoing CTs. ${ }^{1}$ In an initial study, the CT image quality was conclusive. The main lesions could be identified in all patient cases that led to clinical key decisions and focused treatment. ${ }^{5}$ Therefore, the authors concluded that the use of $\mathrm{mCPR}$ devices is feasible during image acquisition. ${ }^{5}$

Although several trials have been made, it cannot be proven that humans benefit from mechanical chest compressions. ${ }^{15}$ Also, targeted post-ROSC CT has not been adopted in the American Heart Association ACLS 2010 guidelines or by the International Liaison Committee on Resuscitation (ILCOR).

\section{CONCLUSION}

This case report shows an interesting approach to outof-hospital cardiac arrest (OHCR) caused by pulmonary embolism in both diagnoses and treatment. Contrast-enhanced CT imaging with ongoing automated chest compressions is feasible within 30 minutes after admission.

Competing Interests: None declared.

\section{REFERENCES}

1. Deakin CD, Nolan JP, Soar J, et al. European Resuscitation Council guidelines for resuscitation 2010 section 4. Adult advanced life support. Resuscitation 2010;81(10):1305-52.
2. Murphy J, Mulvihill N, Mulcahy D, et al. Percutaneous catheter and guidewire fragmentation with local administration of recombinant tissue plasminogen activator as a treatment for massive pulmonary embolism. Eur Rad 1999; 9(5):959-64.

3. Larsen MP, Eisenberg MS, Cummins RO, et al. Predicting survival from out-of-hospital cardiac arrest: a graphic model. Ann Emerg Med 1993;22(11):1652-8.

4. Stiell I, Brown S, Christenson J, et al. What is the role of chest compression depth during out-of-hospital cardiac arrest resuscitation? Crit Care Med 2012;40(4):1192.

5. Wirth S, Körner M, Treitl ML, et al. Computed tomography during cardiopulmonary resuscitation using automated chest compression devices_-an initial study. Eur Rad 2009;19(8):1857-66.

6. Huber-Wagner S, Lefering R, Qvick L-M, et al. Effect of whole-body CT during trauma resuscitation on survival: a retrospective, multicentre study. Lancet 2009;373(9673): 1455-61.

7. Spöhr F, Arntz H, Bluhmki E, et al. International multicentre trial protocol to assess the efficacy and safety of tenecteplase during cardiopulmonary resuscitation in patients with out-of-hospital cardiac arrest: the Thrombolysis in Cardiac Arrest (TROICA) Study. Eur 7 Clin Invest 2005; 35(5):315-23.

8. Bottiger BW, Arntz H-R, Chamberlain DA, et al. Thrombolysis during resuscitation for out-of-hospital cardiac arrest. NE7M 2008;359(25):2651.

9. Scholz K, Hilmer T, Schuster S, et al. Thrombolysis in resuscitated patients with pulmonary embolism. Deutsche medizinische Wochenschrift (1946) 1990;115(24):930.

10. Abella BS, Sandbo N, Vassilatos P, et al. Chest Compression Rates During Cardiopulmonary Resuscitation Are Suboptimal A Prospective Study During In-Hospital Cardiac Arrest. Circulation 2005;111(4):428-34.

11. Wik L, Kramer-Johansen J, Myklebust H, et al. Quality of cardiopulmonary resuscitation during out-of-hospital cardiac arrest. FAMA 2005;293(3):299-304.

12. Chelly J, Mongardon N, Dumas F, et al. Benefit of an early and systematic imaging procedure after cardiac arrest: Insights from the PROCAT (Parisian Region Out of Hospital Cardiac Arrest) registry. Resuscitation 2012;83(12):1444-50.

13. Kanz K-G, Huber-Wagner S, Kay M, et al. Feasibility of focused assessment with computed tomography in resuscitation (FACTR). Resuscitation 2008;77:S44.

14. Moriwaki Y, Tahara Y, Kosuge T, et al. Etiology of out-ofhospital cardiac arrest diagnosed via detailed examinations including perimortem computed tomography. 7 Emerg Trauma Shock 2013;6(2):87.

15. Abella BS. The importance of cardiopulmonary resuscitation quality. Curr Opin Crit Care 2013;19(3):175-80. 